\title{
Sur les points périodiques des applications rationnelles en dynamique ultramétrique
}

\author{
par \\ Jean-Paul Bézivin (Caen)
}

I. Introduction, notations et résultats. Il y a eu récemment des travaux originaux et intéressants sur les problèmes de dynamique des fractions rationnelles en une variable en analyse ultramétrique, c'est-à-dire quand le corps de base est un corps ultramétrique $K$. On définit, et on s'intéresse dans ce cadre aux propriétés des ensembles de Fatou et de Julia associées à une fraction rationnelle à coefficients dans $K$. Parmi les articles de base sur le sujet, on renvoie les lecteurs aux articles de la bibliographie, en particulier aux articles de L. Hsia et R. Benedetto [HS1, HS2, BE1, BE2, BE3, BE4].

Pour ce qui concerne la théorie complexe, on pourra consulter le livre récent de J. Milnor [MI] ou le livre de A. F. Beardon [BEA].

Nous allons commencer par donner quelques définitions. Soient $p$ un nombre premier rationnel fixé, et $\mathbb{C}_{p}$ le complété d'une clôture algébrique du corps $\mathbb{Q}_{p}$ des nombres $p$-adiques. Pour un polynôme $H$ à coefficients dans $\mathbb{C}_{p}$, on note $d(H)$ son degré. Soit $R$ une fraction rationnelle; on écrit $R=P / Q$, avec $P$ et $Q$ polynômes premiers entre eux; on notera alors $d(R)$ la quantité $\max \{d(P), d(Q)\}$, qui sera le degré de $R$.

On supposera dans toute la suite que $R$ est de degré au moins 2 .

La fraction rationnelle $R$ définit une application de $\mathbb{P}^{1}\left(\mathbb{C}_{p}\right)$ dans luimême, que nous visualiserons simplement en rajoutant un point à l'infini à $\mathbb{C}_{p}$.

Dans ce cadre, on peut itérer la transformation qui à $x \in \mathbb{C}_{p} \cup\{\infty\}$ associe $R(x)$; nous noterons $R^{[n]}$ les itérées successives de $R$.

On peut définir une distance sur $\mathbb{P}^{1}\left(\mathbb{C}_{p}\right)$, la distance sphérique, de la façon suivante : pour deux points $A=(x, y)$ et $B=(u, v)$, on pose

$$
\Delta(A, B)=\frac{|v x-u y|}{\max \{|x|,|y|\} \max \{|u|,|v|\}} .
$$

2000 Mathematics Subject Classification: Primary 11S, 37E. 
On pourra voir l'article [BE1] de R. Benedetto pour les propriétés de cette distance.

On définit, de manière analogue au cas où le corps de base est le corps des nombres complexes, l'ensemble de Fatou $\mathcal{F}(R)$ de la fraction rationnelle $R$ comme étant l'ensemble des points de $\mathbb{P}^{1}\left(\mathbb{C}_{p}\right)$ où la famille $R^{[n]}$ des itérées de $R$ est équicontinue, c'est-à-dire des points $x \in \mathbb{P}^{1}\left(\mathbb{C}_{p}\right)$ tels qu'il existe un voisinage $V$ de $x$ pour la distance $\Delta$ tel que, pour tout $\varepsilon>0$, il existe $\delta>0$ tel que pour tout couple $(a, b)$ d'éléments de $V$ vérifiant $\Delta(a, b)<\delta$, on ait $\Delta\left(R^{[n]}(a), R^{[n]}(b)\right)<\varepsilon$.

Il est clair, d'après la définition, que l'ensemble de Fatou de $R$ est un ouvert. Le complémentaire de l'ensemble de Fatou est donc une partie fermée de $\mathbb{P}^{1}\left(\mathbb{C}_{p}\right)$ que l'on appelle l'ensemble de Julia $\mathcal{J}(R)$ de $R$.

Soit $x \in \mathbb{P}^{1}\left(\mathbb{C}_{p}\right)$. On appelle orbite de $x$ sous l'action de $R$ l'ensemble $\left\{R^{[n]}(x): n \in \mathbb{N}\right\}$.

L'ensemble de toutes les pré-images des éléments de l'orbite de $x$ est appelée la grande orbite de $x$.

Des points intéressants pour l'étude des ensembles de Fatou et de Julia sont les points fixes et périodiques, et pré-périodiques de $R$.

Un point fixe de $R$ est un point $x \in \mathbb{P}^{1}\left(\mathbb{C}_{p}\right)$ vérifiant $R(x)=x$, un point périodique un point $x$ tel qu'il existe un entier $n \geq 1$ tel que $R^{[n]}(x)=x$; dans ce cas, le plus petit de ces entiers $n$ est la période de $x$.

Un point pré-périodique est une pré-image d'un point périodique de $R$.

Si $x$ est un point périodique de $R$, qui n'est pas le point à l'infini, on définit son multiplicateur comme étant $\lambda=\left(R^{[n]}\right)^{\prime}(x)$. On vérifie immédiatement qu'en conjuguant $R$ par une homographie, on trouve une nouvelle fraction rationnelle telle que l'image de $x$ soit encore un point périodique de même période, et si l'image de $x$ n'est pas $\infty$, de même multiplicateur. Ceci permet de définir le multiplicateur de $\infty$ quand celui-ci est un point périodique de $R$, en l'envoyant par une homographie sur un point à distance finie.

On dira que le point périodique $x$ de $R$ est :

(a) Un point attractif si son multiplicateur $\lambda$ est de module $<1$.

(b) Un point neutre si son multiplicateur $\lambda$ est de module 1.

(c) Un point répulsif si son multiplicateur $\lambda$ est de module $>1$.

Un résultat qui nous sera utile plus tard est que, si $d$ est un point périodique de $R$, de période $m$, et si on prend $k \in \mathbb{N}$, le point $R^{[k]}(d)$, qui est l'un des $m$ points dans l'orbite de $d$, admet le même multiplicateur que $d$ (tous les points de l'orbite de $d$ ont même multiplicateur); on a

$$
\left(R^{[m]}\right)^{\prime}(d)=\left(R^{[m]}\right)^{\prime}\left(R^{[k]} d\right) .
$$

On a la proposition suivante : 
THÉORÈme 1. Soient $R$ une fraction rationnelle et $x$ un point périodique de R. Alors :

(a) Si $x$ est attractif ou neutre, alors $x$ est dans l'ensemble de Fatou.

(b) Si $x$ est répulsif, alors $x$ est dans l'ensemble de Julia.

Preuve. Voir [BE2, Proposition 1.1].

Contrairement à ce qui se passe en analyse complexe, l'ensemble de Julia d'une fraction rationnelle $R$ peut, en analyse ultramétrique, être vide. Par contre, l'ensemble de Fatou n'est jamais vide :

ThÉORÈme 2. Soit $R$ une fraction rationnelle. Alors $R$ possède au moins un point fixe neutre ou attractif; par suite son ensemble de Fatou n'est jamais vide.

Preuve. Voir [BE2, Corollary 1.3].

Il est bien clair, d'après ce qui précède, que s'il existe un point périodique répulsif, il est dans l'ensemble de Julia, et donc celui-ci est non vide.

Il se posait donc la question de voir le rapport entre l'ensemble des points périodiques répulsifs et l'ensemble de Julia de $R$.

En analyse complexe, dans la même situation, l'ensemble de Julia est l'adhérence des points périodiques répulsifs en raison notamment du fait que l'ensemble des points périodiques attractifs ou neutres est fini.

Une question posée par L. Hsia dans [HS1, Question 1, page 303] est d'examiner si cette propriété est vraie aussi en analyse ultramétrique.

Nous allons donner dans cet article une réponse partiellement positive à cette question :

ThÉORÈme 3. Soit $R$ une fraction rationnelle, de degré au moins 2. On suppose que $R$ possède au moins un point périodique répulsif. Alors l'ensemble de Julia de $R$ est l'adhérence de l'ensemble des points périodiques répulsifs. De plus, tout point de l'ensemble de Julia est point d'accumulation de points périodiques répulsifs.

Par contre, notre méthode de démonstration ne nous permet pas de montrer que si la fraction rationnelle $R$ n'a pas de points périodiques répulsifs, alors son ensemble de Julia est vide, ce qui est aussi une question posée par L. Hsia dans [HS1, question 4, page 303], et qui compléterait le résultat précédent, en montrant que l'ensemble de Julia d'une fraction rationnelle $R$ est dans tous les cas l'adhérence de l'ensemble des points périodiques répulsifs de $R$.

La méthode de démonstration de notre résultat principal ressemble à celle utilisée par Julia dans [JU], avec la différence que, en général, les arguments utilisés en analyse complexe passent mal en analyse ultramétrique. 
L'auteur remercie vivement le referee pour une lecture attentive du texte original, et en particulier pour une simplification de la fin de la preuve du résultat principal.

II. Rappels de résultats. On a tout d'abord des résultats élémentaires sur les ensembles de Julia et de Fatou :

Proposition 1. Soit $R$ une fraction rationnelle. Alors les ensembles de Julia et de Fatou sont complètement invariants par $R$, c'est-à-dire que les images directes et réciproques d'un élément de ces ensembles sont encore dans l'ensemble en question.

Preuve. Cela vient simplement du fait que les ensembles de Julia et Fatou sont stables sous l'action de $R$, et ils sont complémentaires.

Proposition 2. Soient $R$ une fraction rationnelle, $m \geq 1$ un entier et $S(x)=R^{[m]}(x)$ l'itérée d'ordre $m$ de $R$. Alors les ensembles de Julia et de Fatou de $S$ sont les mêmes que ceux de $R$.

Un outil très utile est le critère de Hsia, analogue d'un résultat de Montel [HS2] :

CRITÈre DE HsIA. Soit $F$ une famille de séries entières convergentes sur un disque $D$ de $\mathbb{P}^{1}\left(\mathbb{C}_{p}\right)$. On suppose que l'image de $D$ par les éléments $f \in F$ est inclus dans un ensemble Yindépendant de $f$ dont le complémentaire contient au moins deux points de $\mathbb{P}^{1}\left(\mathbb{C}_{p}\right)$. Alors la famille $F$ est équicontinue sur D.

Preuve. Nous donnons une preuve pour la commodité du lecteur. Comme $D$ est un disque de $\mathbb{P}^{1}\left(\mathbb{C}_{p}\right)$, on se ramène d'abord, par conjugaison par une homographie, au cas où c'est un disque de $\mathbb{C}_{p}$, que nous notons $B(a, r)$; ce disque peut être ouvert ou fermé, nous traitons le cas du disque ouvert en laissant celui du disque fermé au lecteur. Soit $b \notin Y, b \neq \infty$, qui existe car le complémentaire de $Y$ a au moins deux points. On peut écrire tout $f \in F$ comme une série entière $f(x)=a_{0}+a_{1}(x-a)+\ldots+a_{k}(x-a)^{k}+\ldots$ qui converge sur $D$. On sait que $f(x) \neq b$ pour tout $x$ dans $D$. Il en résulte que pour tout $x \in D$, on a $|f(x)-b|=|f(a)-b|$ et que $\left|a_{k}\right| \varrho^{k}<\left|a_{0}-b\right|$ pour tout $k \geq 1$ et $\varrho<r$. Si on prend $x, y \in D$, il vient alors que

$f(x)-f(y)=(x-y) \sum_{k \geq 1} a_{k}\left((x-a)^{k-1}+(x-a)^{k-2}(y-a)+\ldots+(y-a)^{k-1}\right)$.

En posant $\varrho=\max \{|x-a|,|y-a|\}<r$, il vient que $|f(x)-f(y)| \leq$ $|x-y| \max \left\{\left|a_{k}\right| \varrho^{k-1}\right\}$; donc on a $|f(x)-f(y)| \leq|x-y| \max \left\{\left|a_{k}\right| r^{k-1}\right\}$ et donc

$$
|f(x)-f(y)| \leq|x-y| \frac{|f(a)-b|}{r} .
$$


On doit maintenant revenir à la distance sphérique et discuter. La distance sphérique entre $f(x)$ et $f(y)$ est

$$
\Delta(f(x), f(y))=\frac{|f(x)-f(y)|}{\max \{|f(x)|, 1\} \max \{|f(y)|, 1\}} .
$$

On discute suivant la position de $f(a): \operatorname{si}|f(a)-b| \leq \max \{|b|, 1\}$, il vient que $|f(a)| \leq \max \{|b|, 1\}$, et comme $\max \{|f(x)|, 1\} \geq 1$ et $\max \{|f(y)|, 1\} \geq 1$, on a

$$
\Delta(f(x), f(y)) \leq \frac{\max \{|b|, 1\}}{r}|x-y| .
$$

Si maintenant $|f(a)-b|=|f(x)-b|=|f(y)-b|>\max \{|b|, 1\}$, alors $|f(a)|=|f(x)|=|f(y)|>1$, et il vient

$$
\Delta(f(x), f(y)) \leq \frac{1}{r}|x-y|
$$

Il existe donc une constante $c>0$ telle que, pour tous $x, y \in D$ et $f \in$ $F$, on ait $\Delta(f(x), f(y)) \leq c|x-y|$ et donc une constante $C$ telle que $\Delta(f(x), f(y)) \leq C \Delta(x, y)$, puisque les deux distances sont uniformément équivalentes sur $D$, ce qui termine la démonstration.

En fait, il existe, comme en analyse complexe, une généralisation immédiate (cf. [BEA, page 57]) :

ThÉorÈme 4 . Soient $D$ un disque de $\mathbb{P}^{1}\left(\mathbb{C}_{p}\right)$ et $F$ une famille de séries entières convergentes sur $D$. On suppose que, pour tout $f \in F$, il existe deux points distincts $a_{f}, b_{f}$ dans $\mathbb{C}_{p}$ tels que

(a) l'image $f(D)$ de $D$ par $f$ est incluse dans $Y_{f}=\mathbb{P}^{1}\left(\mathbb{C}_{p}\right)-\left\{a_{f}, b_{f}\right\}$;

(b) il existe $m>0$ tel que $\Delta\left(a_{f}, b_{f}\right) \geq m$ pour tout $f \in F$.

Alors $F$ est équicontinue.

Preuve. Soit $h_{f}$ une homographie non triviale de la forme

$$
h_{f}(x)=\frac{a x+b}{c x+d},
$$

qui envoie 0 sur $a_{f}$ et $\infty$ sur $b_{f}$. On peut supposer que $a, b, c, d$ sont tous dans l'anneau d'entiers de $\mathbb{C}_{p}$, l'un d'entre eux étant de module 1 . Il y a une infinité de telles fractions rationnelles, et si on suppose que $a_{f}, b_{f}$ sont dans $\mathbb{C}_{p}$ (les cas restant sont laissés au lecteur), elles sont données par les relations $b=d a_{f}$ et $a=c b_{f}$. La condition sur $a, b, c, d$ est respectée si on prend

$$
|c|=\min \left\{1, \frac{1}{\left|b_{f}\right|}\right\}=\frac{1}{\max \left\{1,\left|b_{f}\right|\right\}}
$$

et de même

$$
|d|=\min \left\{1, \frac{1}{\left|a_{f}\right|}\right\}=\frac{1}{\max \left\{1,\left|a_{f}\right|\right\}} .
$$


Pour une fraction rationnelle $h(x)=(a x+b) /(c x+d)$ quelconque, avec $a d-b c \neq 0$, on a la relation

$$
\Delta(h(x), h(y)) \leq \frac{\Delta(x, y)}{|a d-b c|} .
$$

Ceci donne ici pour $|a d-b c|$ la quantité $|d| \cdot|c| \cdot\left|a_{f}-b_{f}\right|=\Delta\left(a_{f}, b_{f}\right)$.

Il vient donc que

$$
\Delta\left(h_{f}(x), h_{f}(y)\right) \leq \frac{\Delta(x, y)}{\Delta\left(a_{f}, b_{f}\right)} \leq \frac{\Delta(x, y)}{m} .
$$

On considère maintenant la famille des applications de la forme $g_{f}=$ $h_{f}^{[-1]} \circ f$. Ce sont des séries convergentes dans le disque $D$, car l'unique pôle de $h_{f}^{[-1]}$ est en $b_{f}$, qui n'est pas dans l'image de $f$. D'autre part, $g_{f}$ évite les deux valeurs 0 et $\infty$, par construction. Le théorème précédent s'applique, et montre que la famille des $g_{f}, f \in F$, est équicontinue sur $D$. Soit $\varepsilon>0$; il existe $\delta>0$ tel que si $x, y \in D$ et si $\Delta(x, y)<\delta$, on a $\Delta\left(g_{f}(x), g_{f}(y)\right)<\varepsilon$ pour tout $f \in F$. D'autre part, on a $f=h_{f} \circ g_{f}$, et par suite si $\Delta(x, y)<\delta$, on a

$$
\Delta(f(x), f(y))=\Delta\left(h_{f} \circ g_{f}(x), h_{f} \circ g_{f}(y)\right) \leq \frac{\Delta\left(g_{f}(x), g_{f}(y)\right)}{m} \leq \frac{\varepsilon}{m},
$$

ce qui démontre l'équicontinuité de la famille $F$, et termine la démonstration.

Nous allons maintenant utiliser une méthode introduite par Poincaré dans le cas où le corps de base est $\mathbb{C}$, qui permet de montrer l'existence d'une fonction méromorphe dans tout $\mathbb{C}_{p}$ qui permet de décrire convenablement les points périodiques de $R$.

Lemme 1. Soit $R$ une fraction rationnelle, nulle à l'origine, telle que $R^{\prime}(0)=q$ est de module $|q|>1$, et envoyant le point à l'infini sur lui-même. Alors il existe une fonction méromorphe $\Phi$ telle que $\Phi(z)=z+\ldots$ dans un voisinage de 0 , vérifiant $\Phi(q z)=R(\Phi(z))$.

Preuve. On écrit $R=P / Q$, en supposant que $Q(0)=1$ et $P(0)=0$, $P^{\prime}(0)=q$, et $P, Q$ premiers entre eux.

On pose

$$
R^{*}(u, v)=R\left(\frac{u}{v}\right)=\frac{P^{*}(u, v)}{Q^{*}(u, v)} ;
$$

en raison des hypothèses faites, le degré $s$ de $P$ est strictement supérieur au degré $t$ de $Q$. On a $P^{*}(u, v)=q u v^{s-1}+\alpha_{2} u^{2} v^{s-2}+\ldots+\alpha_{s} u^{s}$ et $Q^{*}(u, v)=$ $v^{s}+\beta_{1} u v^{s-1}+\ldots+\beta_{t} u^{t} v^{s-t}$. On étudie le système de deux équations : $\phi(q z)=P^{*}(\phi(z), \psi(z))$ et $\psi(q z)=Q^{*}(\phi(z), \psi(z))$; on veut montrer que ce système admet un couple $(\phi, \psi)$ de solutions fonctions entières dans $\mathbb{C}_{p}$.

Dans la suite, si $h$ est une série entière, on note $C_{n}(h)$ le coefficient de $z^{n}$ dans $h$, et $h_{n}$ le polynôme $\sum_{k=0}^{n} C_{k}(h) z^{k}$. 
Nous imposons que $\phi(z)=z+\ldots=\sum_{k=1}^{+\infty} a_{k} z^{k}$ avec $a_{0}=0, a_{1}=1$, et $\psi(z)=\sum_{k=0}^{+\infty} b_{k} z^{k}$ avec $b_{0}=1$.

Ceci étant posé, les relations fonctionnelles permettent déjà de définir deux uniques séries formelles solutions du système.

En effet, le coefficient de $z^{n}$ dans $P^{*}(\phi(z), \psi(z))$ est de la forme $q C_{n}\left(\phi \psi^{s-1}\right)+\alpha_{2} C_{n}\left(\phi^{2} \psi^{s-2}\right)+\ldots+\alpha_{s} C_{n}\left(\phi^{s}\right)$ (nous rappelons à ce propos que nous notons $\phi^{k}, \psi^{l}$ les puissances ordinaires de $\phi$ et $\psi$ ).

Soit $k$ un entier, $k \geq 1$. Alors les coefficients de $z^{j}, j \leq n$, dans $\phi^{k}$ ne font intervenir que les coefficients de $z^{m}, m \leq n-k+1$, dans $\phi$. De même, dans le produit $\phi^{k} \psi^{s-k}$, les coefficients de $z^{j}, j \leq n$, dans $\psi^{s-k}$ ne font intervenir que les coefficients de $z^{m}, m \leq n-k$, dans $\psi$, car $z^{k}$ est en facteur dans $\phi^{k}$.

On a donc la formule $C_{n}\left(\phi^{k} \psi^{s-k}\right)=C_{n}\left(\phi_{n-k+1}^{k} \psi_{n-k}^{s-k}\right)$. Il en résulte

$$
a_{n} q^{n}=q C_{n}\left(\phi_{n} \psi_{n-1}^{s-1}\right)+\alpha_{2} C_{n}\left(\phi_{n-1}^{2} \psi_{n-2}^{s-2}\right)+\ldots+\alpha_{s} C_{n}\left(\phi_{n-s}^{s}\right) .
$$

En particulier, le coefficient $a_{n}$ n'intervient au second membre que dans le premier terme, qui est de la forme

$$
q C_{n}\left(\phi_{n} \psi_{n-1}^{s-1}\right)=q a_{n}+q C_{n}\left(\phi_{n-1} \psi_{n-1}^{s-1}\right)
$$

donc

$$
a_{n}\left(q^{n}-q\right)=q C_{n}\left(\phi_{n-1} \psi_{n-1}^{s-1}\right)+\alpha_{2} C_{n}\left(\phi_{n-1}^{2} \psi_{n-2}^{s-2}\right)+\ldots+\alpha_{s} C_{n}\left(\phi_{n-s}^{s}\right) .
$$

Puisque $n>1$, le coefficient $q^{n}-q$ est non nul, ce qui permet donc d'exprimer $a_{n}$ en fonction des $a_{k}, b_{k}$ avec $k<n$. On fait le même travail pour la seconde équation :

$$
b_{n} q^{n}=C_{n}\left(\psi^{s}\right)+\beta_{1} C_{n}\left(\phi \psi^{s-1}\right)+\ldots+\beta_{t} C_{n}\left(\phi^{t} \psi^{s-t}\right) .
$$

On a encore

$$
b_{n} q^{n}=C_{n}\left(\psi_{n}^{s}\right)+\beta_{1} C_{n}\left(\phi_{n} \psi_{n-1}^{s-1}\right)+\ldots+\beta_{t} C_{n}\left(\phi_{n-t+1}^{t} \psi_{n-t}^{s-t}\right) .
$$

Modulo $z^{n+1}$, on a $\psi_{n}^{s}$ congru à $\psi_{n-1}^{s}+s b_{n} z^{n}$, et comme $\psi(0)=1$, on a aussi $C_{n}\left(\phi_{n} \psi_{n-1}^{s-1}\right)=a_{n}+C_{n}\left(\phi_{n-1} \psi_{n-1}^{s-1}\right)$, donc

$$
b_{n}\left(q^{n}-s\right)=\beta_{1} a_{n}+C_{n}\left(\psi_{n-1}^{s}\right)+\beta_{1} C_{n}\left(\phi_{n-1} \psi_{n-1}^{s-1}\right)+\ldots+\beta_{t} C_{n}\left(\phi_{n-t+1}^{t} \psi_{n-t}^{s-t}\right) .
$$

On remarquera que $q^{n}-s$ est non nul, car $|q|^{n}>1$ si $n \geq 1$, et comme $s$ est entier, on a $|s| \leq 1$.

Ces formules permettent de définir de manière unique deux suites $a_{n}$ et $b_{n}$ compte tenu des valeurs initiales $a_{0}=0, a_{1}=1$ et $b_{0}=1$; on a donc un unique couple de séries formelles solution.

Nous allons montrer maintenant que le rayon de convergence de ces deux séries est non nul.

Soient $U=\sum u_{n} z^{n}$ et $V=\sum v_{n} z^{n}$ deux séries entières, et supposons que $\left|u_{n}\right| \leq M_{1} \mu^{n}$ et $\left|v_{n}\right| \leq M_{2} \mu^{n}$ pour tout $n$, où $M_{1}, M_{2}, \mu$ sont des constantes positives. Par la formule donnant le coefficients du terme en $z^{n}$ 
dans le produit $W=\sum w_{n} z^{n}$ de $U$ et de $V$, il est alors immédiat que $\left|w_{n}\right| \leq M_{1} M_{2} \mu^{n}$ pour tout $n$.

Soit $M$ un majorant de $|q|$ et des $\left|\alpha_{j}\right|,\left|\beta_{j}\right|$. Soit $N$ un entier assez grand de façon que $M<|q|^{N}$. Soit enfin $\mu$ un réel positif, assez grand de façon que $\left|a_{j}\right| \leq \mu^{j}$ et $\left|b_{j}\right| \leq \mu^{j}$ pour $j=0, \ldots, N$. Nous allons montrer que $\left|a_{n}\right| \leq \mu^{n}$ et $\left|b_{n}\right| \leq \mu^{n}$ par récurrence sur $n$. Le cas de $n=0, \ldots, N$ étant réglé par les hypothèses faites, nous supposons que $n \geq N+1$, et que le résultat est acquis pour les indices $k \leq n-1$.

Par ce qui précède, il vient, puisque les coefficients d'indice $k$ de $\psi_{n-h}$ et de $\phi_{n-h}$ pour $h$ non nul sont majorés par $\mu^{k}$, l'inégalité

$$
\left|C_{n}\left(\phi_{n-j}^{k} \psi_{n-k}^{s-k}\right)\right| \leq \mu^{n} \quad \text { pour } j \geq 1, k \geq 1 .
$$

Il en résulte d'après la formule donnant $a_{n}\left(q^{n}-q\right)$ que $\left|a_{n}\right| \cdot|q|^{n} \leq M \mu^{n}$, d'où $\left|a_{n}\right| \leq M(\mu /|q|)^{n}$. Puisque $n \geq N+1$, cette dernière quantité est majorée par $\mu^{n}$, ce qui démontre l'assertion.

On fait de même pour $\left|b_{n}\right|$, le raisonnement est exactement le même.

Nous avons donc démontré que les séries formelles $\phi$ et $\psi$ ont un rayon de convergence non nul.

Supposons maintenant que le minimum $r$ de ces rayons de convergence soit fini, de sorte que $r$ est le rayon de convergence de l'une des deux séries, le rayon de convergence de l'autre étant au moins $r$.

On a les formules

$$
\phi(z)=P^{*}\left(\phi\left(\frac{z}{q}\right), \psi\left(\frac{z}{q}\right)\right) \quad \text { et } \quad \psi(z)=Q^{*}\left(\phi\left(\frac{z}{q}\right), \psi\left(\frac{z}{q}\right)\right) .
$$

Les deux expressions au second membre ont pour rayon de convergence au moins $|q| r$. Comme l'une des séries au premier membre a pour rayon de convergence exactement $r$, il y a une contradiction, qui montre que les deux séries $\phi$ et $\psi$ définissent deux fonctions entières dans $\mathbb{C}_{p}$. Il est clair que le quotient $\Phi(z)=\phi(z) / \psi(z)$ est une fonction méromorphe qui vérifie $\Phi(q z)=R(\Phi(z))$, ce qui termine la démonstration.

REMARQue. Les deux fonctions $\phi$ et $\psi$ sont sans zéro commun, en raison de l'hypothèse que $P$ et $Q$ sont premiers entre eux; en effet, si $z_{0}$ est un zéro commun éventuel, il est non nul, et on peut supposer que c'est un zéro commun de module minimal. Alors $z_{0} / q=z_{1}$ n'est pas zéro commun de $\phi$ et $\psi$, et on a

$$
P^{*}\left(\phi\left(z_{1}\right), \psi\left(z_{1}\right)\right)=0=Q^{*}\left(\phi\left(z_{1}\right), \psi\left(z_{1}\right)\right) .
$$

En raison de la forme de $P^{*}$, il en résulte que $\psi\left(z_{1}\right)$ n'est pas nul, et donc $\omega=\phi\left(z_{1}\right) / \psi\left(z_{1}\right)$ est zéro commun de $P$ et $Q$, ce qui est contraire au fait que $P$ et $Q$ sont premiers entre eux.

Lemme 2. Soit $R$ une fraction rationnelle, de degré au moins 2 , telle que l'infini soit un point fixe ainsi que 0 , celui-ci étant répulsif, de multipli- 
cateur $q$. Soit $b$ un élément non nul de $\mathbb{C}_{p}$ tel que $R(b)=0$. Alors il existe une suite de points périodiques répulsifs de $R$, distincts de $b$, et de limite $b$.

Preuve. Par hypothèse, on se trouve dans les conditions d'application du résultat précédent. Soit donc $\Phi(x)=\phi(x) / \psi(x)$ une fonction méromorphe dans $\mathbb{C}_{p}$ tout entier, vérifiant les propriétés du résultat précédent.

1. On montre tout d'abord qu'il existe $c \in \mathbb{C}_{p}$ tel que $\Phi(c)=b$. Si un tel $c$ n'existe pas, la fonction $\phi(x)-b \psi(x)$ est une fonction entière dans $\mathbb{C}_{p}$ qui ne s'annule pas, donc une constante $\lambda$; en remplaçant $x$ par 0 , il vient que $\lambda=-b$, de sorte que $\phi(x)=b \psi(x)-b$. En reprenant les équations qui définissent $\phi$ et $\psi$, il vient $\psi(q x)=Q^{*}(\lambda+b \psi(x), \psi(x))$ et $\lambda+b \psi(q x)=$ $\phi(q x)=P^{*}(\lambda+b \psi(x), \psi(x))$, d'où l'on tire que $\lambda+b Q^{*}(\lambda+b \psi(x), \psi(x))=$ $P^{*}(\lambda+b \psi(x), \psi(x))$. La fonction $\psi(x)$ ne peut être constante, car alors par la relation $\lambda+b \psi(x)=\phi(x)$, la fonction $\phi(x)$ serait constante, donc nulle. Par suite la fonction $\psi(x)$ est surjective, donc on a pour tout $v$ dans $\mathbb{C}_{p}$, $\lambda+b Q^{*}(\lambda+b v, v)=P^{*}(\lambda+b v, v)$. On a maintenant les relations $P^{*}(u, v)=$ $v^{s} P(u / v)$ et $Q^{*}(u, v)=v^{s} Q(u / v)$, il en résulte que

$$
\lambda+b v^{s} Q\left(b+\frac{\lambda}{v}\right)=v^{s} P\left(b+\frac{\lambda}{v}\right) .
$$

Posant $x=b+\lambda / v$, il vient alors que

$$
R(x)=b+(-1)^{s-1} \frac{(x-b)^{s}}{b^{s-1} Q(x)},
$$

ce qui est contradictoire avec $R(b)=0$ et $b \neq 0$.

2. Nous allons maintenant construire une suite de points périodiques de $R$, différents de $b$ et de limite $b$.

On a la relation $\Phi(q z)=R(\Phi(z))$, d'où en remplaçant $z$ par $c$, on obtient que $\Phi(q c)=R(\Phi(c))=R(b)=0$.

Le point $c$ est donc un zéro de $\Phi(q z)$. On considère son développement de Taylor au voisinage de $c$, que l'on écrit $\Phi(q z)=a_{h}(z-c)^{h}+\ldots$, avec $a_{h} \neq 0$, le nombre $h \geq 1$ étant la multiplicité du zéro $c$. Ce développement est convergent dans un disque de centre $c$, et rayon strictement positif. Soit $\varrho>0$ assez petit, tel que sur le disque de centre $c$, rayon $\varrho$, le développement converge, que l'on ait de plus sur ce disque $|\Phi(q z)|=\left|a_{h}\right| \cdot|z-c|^{h}$, et enfin que $\Phi(z)$ n'ait aucun pôle dans ce disque.

Soit $n$ un entier assez grand. On va considérer la fonction

$$
\theta_{n}(x)=\phi(q z) \psi\left(\frac{z}{q^{n-1}}\right)-\phi\left(\frac{z}{q^{n-1}}\right) \psi(q z) .
$$

La suite de fonctions $\theta_{n}(x)$ converge uniformément sur le disque $B^{+}(c, \varrho)$ de centre $c$ et rayon $\varrho$ vers la fonction $\phi(q x)$, qui possède un zéro dans ce 
disque, à savoir $c$. Pour $n$ assez grand, il en sera donc de même pour la fonction $\theta_{n}$.

En remplaçant $z$ par $c$ dans l'égalité donnant $\theta_{n}$, il en résulte que l'on a $\theta_{n}(c)=-\phi\left(c / q^{n-1}\right) \psi(q c) \neq 0$ à partir d'un certain rang, puisque $c \neq 0$, et $\psi(q c) \neq 0$ en raison du fait que $\phi(q c)=0$, et que $\phi$ et $\psi$ n'ont pas de zéros en commun. Il en résulte que si on appelle $c_{n}$ un zéro de $\theta_{n}$ situé dans le disque $B^{+}(c, \varrho)$, on a $c_{n} \neq c$, la quantité $d_{n}=\Phi\left(c_{n}\right)$ est bien définie, ainsi que la quantité $\Phi\left(c_{n} / q^{n-1}\right)$ à partir d'un certain rang. On a aussi $c_{n} \in B^{+}(c, \varrho)$, et le fait que $\theta_{n}\left(c_{n}\right)=0$ implique que $\Phi\left(q c_{n}\right)=\Phi\left(c_{n} / q^{n-1}\right)$, donc

$$
\left|\Phi\left(q c_{n}\right)\right|=\left|a_{h}\right| \cdot\left|c_{n}-c\right|^{h}=\left|\Phi\left(\frac{c_{n}}{q^{n-1}}\right)\right|
$$

et $\Phi\left(c_{n} / q^{n-1}\right) \rightarrow 0$, de sorte que $c_{n} \rightarrow c$.

On a donc du fait que $c_{n} \neq c$, la propriété que $d_{n}=\Phi\left(c_{n}\right) \neq b$ à partir d'un certain rang.

On a de plus

$$
R^{[n]}\left(\Phi\left(c_{n}\right)\right)=\Phi\left(q^{n} c_{n}\right)=R^{[n-1]}\left(\Phi\left(q c_{n}\right)\right)=R^{[n-1]}\left(\Phi\left(\frac{c_{n}}{q^{n-1}}\right)\right)=\Phi\left(c_{n}\right),
$$

de sorte que $d_{n}=\Phi\left(c_{n}\right)$ est un point périodique de $R$.

3 . Il suffit maintenant démontrer que $d_{n}$ est répulsif pour conclure.

On a $|\Phi(z)|=|z|$ si $|z|$ est assez petit, de sorte que puisque $c_{n} \rightarrow c \neq 0$, donc $c_{n} / q^{n-1} \rightarrow 0$, on a, pour $n$ assez grand,

$$
\left|\Phi\left(\frac{c_{n}}{q^{n-1}}\right)\right|=\frac{\left|c_{n}\right|}{|q|^{n-1}}=\frac{|c|}{|q|^{n-1}} .
$$

Il en résulte que

$$
\left|c_{n}-c\right|=\frac{1}{|q|^{(n-1) / h}}\left(\frac{|c|}{\left|a_{h}\right|}\right)^{1 / h} .
$$

L'égalité $\Phi\left(q^{n} z\right)=R^{[n]}(\Phi(z))$ implique $q^{n} \Phi^{\prime}\left(q^{n} z\right)=\Phi^{\prime}(z)\left(R^{[n]}\right)^{\prime}(\Phi(z))$. En prenant $z=c_{n} / q^{n-1}$, il vient

$$
q^{n} \Phi^{\prime}\left(q c_{n}\right)=\Phi^{\prime}\left(\frac{c_{n}}{q^{n-1}}\right)\left(R^{[n]}\right)^{\prime}\left(\Phi\left(\frac{c_{n}}{q^{n-1}}\right)\right) .
$$

Comme $\Phi\left(q c_{n}\right)=\Phi\left(c_{n} / q^{n-1}\right)$, ceci implique en se souvenant que $d_{n}=\Phi\left(c_{n}\right)$, et que donc $\Phi\left(q c_{n}\right)=R\left(d_{n}\right)$,

$$
q^{n} \Phi^{\prime}\left(q c_{n}\right)=\Phi^{\prime}\left(\frac{c_{n}}{q^{n-1}}\right)\left(R^{[n]}\right)^{\prime}\left(R\left(d_{n}\right)\right) .
$$

On a $\Phi^{\prime}(z)=1+\ldots$, de sorte que si $n$ est assez grand, $\left|\Phi^{\prime}\left(c_{n} / q^{n-1}\right)\right|$ est égal à 1 , et il vient donc $|q|^{n}\left|\Phi^{\prime}\left(q c_{n}\right)\right|=\left|\left(R^{[n]}\right)^{\prime}\left(R\left(d_{n}\right)\right)\right|$. 
D'autre part on a

$$
q \Phi^{\prime}(q z)=h a_{h}(z-c)^{h-1}+\ldots
$$

et par suite si $n$ est assez grand,

$$
|q| \cdot\left|\Phi^{\prime}\left(q c_{n}\right)\right|=|h| \cdot\left|a_{h}\right| \cdot\left|c_{n}-c\right|^{h-1} .
$$

Avec l'estimation déjà vue de $\left|c_{n}-c\right|$, cela donne

$$
|q|^{n}\left|\Phi^{\prime}\left(q c_{n}\right)\right|=|h| \cdot\left|a_{h}\right|^{1 / h}|c|^{(h-1) / h}|q|^{(n-1) / h}=\left|\left(R^{[n]}\right)^{\prime}\left(R\left(d_{n}\right)\right)\right| .
$$

Il en résulte en particulier que $\left|\left(R^{[n]}\right)^{\prime}\left(R\left(d_{n}\right)\right)\right|>1$ pour $n$ assez grand.

Soit maintenant $m_{n}$ la période du point périodique $R\left(d_{n}\right)$, qui est un diviseur de $n$; on écrit $n=l_{n} m_{n}$ et on a facilement que $\left(R^{\left[m_{n}\right]}\right)^{\prime}\left(R\left(d_{n}\right)\right)^{l_{n}}=$ $\left(R^{[n]}\right)^{\prime}\left(R\left(d_{n}\right)\right)$. Par le résultat évoqué dans l'introduction après la définition du multiplicateur, le point $d_{n}$ est dans l'orbite du point périodique $R\left(d_{n}\right)$, et donc on a $\left(R^{\left[m_{n}\right]}\right)^{\prime}\left(d_{n}\right)=\left(R^{\left[m_{n}\right]}\right)^{\prime}\left(R\left(d_{n}\right)\right)$, ce qui montre que $d_{n}$ est un point périodique répulsif.

Puisque $d_{n}=\Phi\left(c_{n}\right) \neq 0$, il est non nul, et il est aussi proche de $b$ que l'on veut, mais distinct de $b$, et $d_{n}=\Phi\left(c_{n}\right) \rightarrow b$ puisque $c_{n} \rightarrow c$; ceci termine la démonstration.

III. Preuve du théorème 3. On se propose donc de montrer que si l'ensemble $\mathcal{L}$ des points périodiques répulsifs de $R$ n'est pas vide, alors l'ensemble de Julia est l'adhérence de $\mathcal{L}$, ou plus précisément que tout point de l'ensemble de Julia de $R$ est point d'accumulation de points périodiques répulsifs de $R$.

1. Nous allons tout d'abord nous ramener au cas où l'origine est un point fixe répulsif, et l'infini un point fixe dans l'ensemble de Fatou pour $R$.

Tout d'abord, étant donné un entier $m \geq 1$, nous avons déjà vu que les ensembles de Julia de $R$ et de $R^{[m]}$ étaient les mêmes. Cette propriété est aussi vraie pour les ensembles $\mathcal{L}(R)$ et $\mathcal{L}\left(R^{[m]}\right)$ des points périodiques répulsifs pour $R$ et $R^{[m]}$; en effet, dire qu'un point $a$ est périodique pour $R$ équivaut à dire qu'il l'est pour $R^{[m]}$; comme un point périodique est répulsif si et seulement si il est dans l'ensemble de Julia, on a bien que $\mathcal{L}(R)=\mathcal{L}\left(R^{[m]}\right)$.

Soit donc $a$ un point périodique répulsif pour $R$, de période $m \geq 1$; on peut donc remplacer $R$ par $R^{[m]}$, et supposer que $a$ est un point fixe répulsif de $R$. Quitte à composer par une homographie, on peut supposer aussi que le point à l'infini est un point fixe dans l'ensemble de Fatou, et le point $a$ à l'origine, ce que nous faisons désormais.

2. Il existe maintenant $b \in \mathbb{C}_{p}$ tel que $R(b)=0$ et $b \neq 0$. En effet, si ce n'est pas le cas, l'équation $R(x)=0$ a une unique racine $x=0$ dans $\mathbb{C}_{p}$; comme le point à l'infini est un point fixe de $R=P / Q$, le polynôme $P$ est 
de degré au moins 2 , donc a une racine multiple en $x=0$. Ceci implique que $R^{\prime}(0)=0$, contredisant le fait que 0 est un point fixe répulsif.

Le polynôme $P(x)-b Q(x)$ ne peut être constant, car le degré de $P$ est supérieur au degré de $Q$ (puisque l'infini est point fixe), donc égal au degré de $R$. Donc il existe $b^{\prime}$ dans $\mathbb{C}_{p}$ tel que $R\left(b^{\prime}\right)=b$. Les points $b$ et $b^{\prime}$ ne peuvent être périodiques, en particulier, on a $b \neq b^{\prime}$.

3. Soient maintenant $x \in \mathcal{J}(R)$ (donc $x \in \mathbb{C}_{p}$ ) et $\varepsilon>0$. Nous allons montrer qu'il existe $y \in \mathcal{L}$ tel que $y \neq x$ et $|y-x|<\varepsilon$.

Il existe $n \in \mathbb{N}^{*}$ et $c$ dans le disque ouvert de centre $x$, rayon $\varepsilon$, tel que $R^{[n]}(c)=b$. En effet, sinon, la famille $R^{[n]}, n \in \mathbb{N}^{*}$, ne prend pas sur ce disque les deux valeurs $b$ et $b^{\prime}$, et par le critère de Hsia, $x$ serait dans l'ensemble de Fatou, ce qui n'est pas.

Soit $S=R^{[n+1]}$. On a $S(c)=0$, et on peut appliquer le lemme 2 : il existe $y$ appartenant à $\mathcal{L}(S)=\mathcal{L}$ tel que $y \neq x$ et $|y-c|<\varepsilon$ (puisqu'il existe une suite de points de $\mathcal{L}$ distincts de $c$ et de limite $c$, il y en aura donc distincts de $x$ ), ce qui implique $|y-x|<\varepsilon$, et ceci termine la démonstration.

\section{Bibliographie}

[BEA] A. F. Beardon, Iteration of Rational Functions, Springer, 1991.

[BE1] R. Benedetto, Reduction, dynamics, and Julia sets of rational functions, J. Number Theory 86 (2001), 175-195.

[BE2] - Hyperbolic maps in p-adic dynamics, Ergodic Theory Dynam. Systems 21 (2001), 1-11.

[BE3] - - p-adic dynamics and Sullivan's no wandering domains theorem, Compositio Math. 122 (2000), 281-298.

[BE4] - Components and periodic points in non-archimedean dynamics, preprint, July 1999.

[HS1] L. Hsia, A weak Néron model with application to p-adic dynamical systems, Compositio Math. 100 (1996), 277-304.

[HS2] -, Closure of periodic points over a non-archimedean field, J. London Math. Soc. 62 (2000), 685-700.

[JU] G. Julia, Mémoire sur l'itération des fractions rationnelles, J. Math. Pures Appl. 8 (1918), 47-245.

[MI] J. Milnor, Dynamics in One Complex Variable. Introductory Lectures, Vieweg, Wiesbaden, 1999.

Département de Mathématiques et Mécanique

Université de Caen

Campus II, Boulevard du Maréchal Juin

BP 5186

14032 Caen Cedex, France

E-mail: Bezivin@math.unicaen.fr 